\title{
Production, purification and characterization of prodigiosin by Serratia nematodiphilia (NCIM 5606) using solid-state fermentation with various substrate
}

\author{
Kamlesh Kumar Maurya , Abhishek Dutt Tripathi, Deepak Kumar* and Suresh Kumar Srivastava** \\ Department of Dairy Science and Food Technology, Institute of Agricultural Sciences, Banaras Hindu University, Varanasi-221005, Uttar Pradesh, India \\ *Department of Microbiology, Institute of Medical Sciences, Banaras Hindu University, Varanasi-221005, Uttar Pradesh, India \\ **School of Biochemical Engineering, Indian Institute of Technology (BHU), Varanasi-221005, Uttar Pradesh, India
}

\section{Article Info}

Article history

Received 10 September 2020

Revised 2 November 2020

Accepted 4 November 2020

Published online 30 December 2020

\section{Keywords}

Prodigiosin

Serratia nematodiphilia

Solid state fermentation

FTIR

\begin{abstract}
Agroindustrial wastes such as wheat bran, rice bran, orange peel, sweet lemon peel and pigeon pea peel were examined in order to choose the substrate that support the maximum prodigiosin yield using Serratia nematodiphilia. The conversion of agrowaste to a valuable prodigiosin by fermentation process is done to minimize the production cost and environmental risks. Findings revealed that the maximum production yield $(1.3075 \mathrm{mg} / \mathrm{l})$ was observed in wheat bran. Sweet lemon and orange peel gave prodigiosin yield of 0.1693 and $0.1495 \mathrm{mg} / \mathrm{l}$, respectively. Pigeon pea and rice bran media gave minimum prodigiosin yield of 0.0082 and $0.0667 \mathrm{mg} / \mathrm{l}$, respectively. Extracted pigment purified by TLC gave Rf value of 0.85 . Further, purified pigment was characterized as prodigiosin by absorption sepctra $(\lambda \max =535 \mathrm{~nm})$ and FTIR.
\end{abstract}

\section{Introduction}

Pigment is the most indispensable trait of any article especially food. Biocolour word comprises of two words bio and color that implies something regular, utilized for pigment reason. Thus, biocolorants can be one of the options in contrast to false quality for expansion into any food material. These are essentially those pigment specialists, which are acquired from the organic sources, for example, plants, animal and microorganisms as well springs of natural color. These natural color are commonly extracted from natural products, vegetables, seeds, roots and microorganisms and are frequently called as 'biocolors' because of their organic source (Sharma, 2014; Rymbai et al., 2011). Lately, look for microorganisms delivering non-poisonous metabolites, has been performed by a few analysts (Downham and Collins, 2000). Further, the waste created from food industry can likewise be one of the substrate for development of these biocolors delivering microorganisms.

Natural colors produced as metabolites by the microorganisms serve as potential substitute for harmful synthetic dyes. Unlike, the conventional colors derived from plants and animal skin, microbial pigment are finding tremendous application in food industries, owing to their easier accessibility, simpler extraction and adequacy. Serratia spp. are gram-negative microbes grouped in the extensive group of Enterobactericeae producing a dark red pigment termed as prodigiosin (Samrot et al., 2011).

Corresponding author: Mr. Kamlesh Kumar Maurya Department of Dairy Science and Food Technology, Institute of Agricultural Sciences, Banaras Hindu University, Varanasi-221005, Uttar Pradesh, India

E-mail: kamleshcfstbhu@gmail.com

Tel.: +91-9794886577

Copyright (c) 2020 Ukaaz Publications. All rights reserved.

Email: ukaaz@yahoo.com; Website: www.ukaazpublications.com
The prodigiosin is natural product and belonging to a family of tripyrrole red color that contains a typical 4-methoxy 2, 2 bipyrrole ring framework. The biosynthesis of the color is a two steps in which mono and bipyrrole antecedents are blended independently and after that gathered to shape prodigiosin (Boger and Patel, 1988). The biosynthesis of the color is a bifurcated procedure in which mono and bipyrrole antecedents are blended independently and after that gathered to shape prodigiosin. Prodigiosin is multifaceted secondary metabolites having chemical formula $\mathrm{C}_{20} \mathrm{H}_{25} \mathrm{~N}_{3} \mathrm{O}$, possessing an unusual structure with three pyrrole rings and a pyrryldipyrrylmethane group comprising three rings where two rings are directly linked to each other, and the third is linked through a methane bridge.

Prodigiosin is produced by numerous microorganism including Serratia marcescens, Vibrio psychroerythrus, Sreptomycin griseoviridis and Hahella chejuensis with antibacterial, antimycotic, immunomodulating, against tumor and antimalarial properties (Frustner, 2003; Giri et al., 2004). Kobayashi and lchikawa (1991) and Matsuyama et al. (1986), deduced that prodigiosin is synthesized as extracellular vesicles or present as intracellular granules in Serratia sp. Colored compounds such as pigments absorb light of characteristic wavelength, determined spectrophotometrically (Cerdeño et al., 2001). Prodigiosin can exist in two different forms depending on the hydrogen ion concentration of the production media. In acidic medium, colored pigment shows characteristic spectra at $535 \mathrm{~nm}$. The pigment production depends on type of fermentation and the cultural conditions. It has been previously reported that SSF shows efficient like high volumetric profitability (Duenas et al., 1995). Agrowaste utilization of compelling use of agroindustrial wastage as substrates that even 
copy the common living surface of organisms and economy (Murugesan et al., 2007) because of its static nature. This reutilization of agrowaste is refreshing with regards to reasonable advancement. In recently SSF has picked up significance in the creation of microbial pigment because of a few monetary favorable circumstances over customary submerged fermentation (Sathya et al., 2009).

In recent years, use of agriculture based product and results acquires significance in bioprocess industries on account of high supplement substance and minimal effort. Changing the waste side-effects to a valuable bioproduct by fermentation diminishes the procedure cost as well as the danger of natural contamination. Customarily, the enhancement is being finished by keeping all parameter consistent and changing different parameters. This does not interaction of one parameter with the other. Our aim is to discover a media that may support the development of the bacteria and in the meantime demonstrate effective to trigger much amounts of color production. In present investigation, we have endeavoured to expand the production of prodigiosin from Serratia nematodiphilia (NCIM 5606) by utilizing wheat bran as well as purified prodigiosin is tested for various activities.

\section{Materials and Methods}

2.1 Microorganism and screening of substrates for prodigiosin production

Bacterial strain Serratia nematodiphilia (NCIM 5606) was procured from the National Collection of Industrial Microorganisms, National Chemical Laboratory, Pune, India. The stock culture was maintained at $28^{\circ} \mathrm{C}$ on nutrient agar slants. Inoculum was prepared by adding a loopful of bacterial colonies in $250 \mathrm{ml}$ conical flasks containing $100 \mathrm{ml}$ of nutrient broth and incubated at $28^{\circ} \mathrm{C}$ for $72 \mathrm{~h}$.

\subsection{Selection of substrate}

Substrates of wheat bran, rice bran, orange peel, sweet lemon peel and pigeon pea peel were examined for their suitability for growth of Serratia nematodiphilia and prodigiosin production.

2.3 Cultivation conditions for prodigiosin production in solidstate fermentation (SSF)

The substrate, wheat bran, rice bran and pigeon pea were obtained from local market mill located in Varanasi and orange peel and sweet lemon peel collected from BHU fruit shop. All substrate were dried at $50^{\circ} \mathrm{C}$, until a constant weight was obtained and then grind with food grinder (Philips) and sieved into particle size of $25-90 \mu \mathrm{m}$. Then, $5 \mathrm{~g}$ of the dried substrate was then placed in a $250-\mathrm{ml}$ of each conical flask and $10 \mathrm{ml}$ of trace metal solution $\mathrm{pH} 7$ added and autoclaved at $121^{\circ} \mathrm{C}$ for $15 \mathrm{~min}$ and cooled to room temperature. Trace metal solution $\mathrm{K}_{2} \mathrm{HPO}_{4}(2 \mathrm{~g} / \mathrm{l})$ and trace ion solution $\left(0.5 \mathrm{~g} / 1 \mathrm{MgSO}_{4} \cdot 7 \mathrm{H}_{2} \mathrm{O}, 0.5 \mathrm{~g} / 1 \mathrm{NaCl}, 0.5 \mathrm{~g} / 1 \mathrm{MnSO}_{4}\right.$, $3.4 \mathrm{mg} / 1, \mathrm{ZnSO}_{4} \cdot 4 \mathrm{H}_{2} \mathrm{O}, 5 \mathrm{mg} / 1 \mathrm{FeSO}_{4} \cdot 7 \mathrm{H}_{2} \mathrm{O}$ and $2 \mathrm{mg} / \mathrm{L} \mathrm{CoCl}_{2} \cdot 6 \mathrm{H}_{2} \mathrm{O}$ were prepared. The flask was inoculated with $1 \mathrm{ml} \mathrm{Serratia}$ nematodiphilia (NCIM 5606) and incubated at $25^{\circ} \mathrm{C}$ for 6 days Figures $1 \mathrm{a}$ and $\mathrm{b}$.

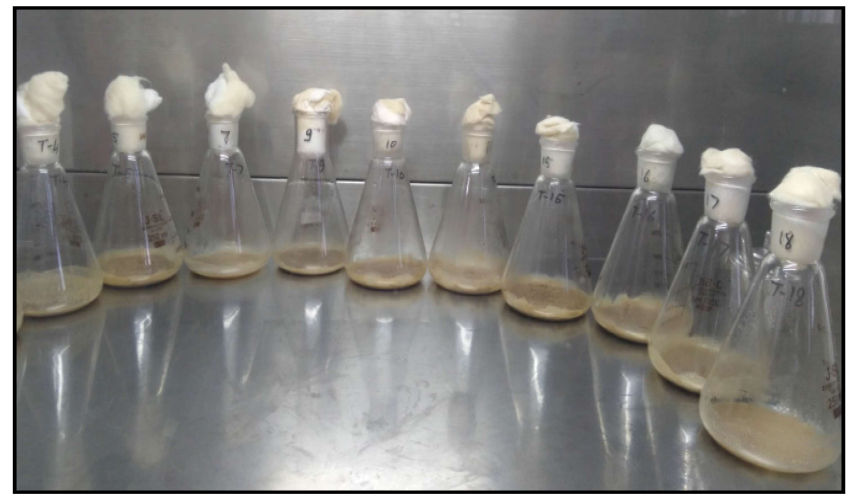

Figure 1a: Before fermentation.

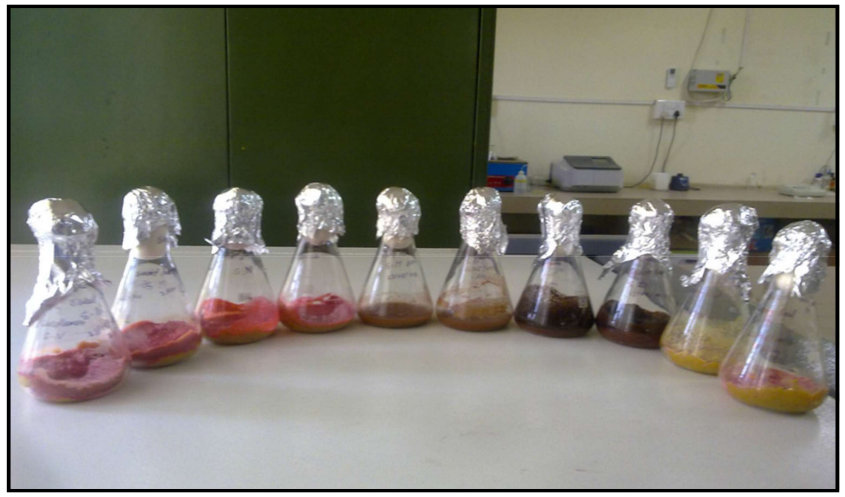

Figure 1b: After fermentation (SSF of different substrate).

\subsection{Extraction and determination of prodigiosin}

First, we add fermented sample with phosphate buffer at pH 7. Then mixed in shaker at $100 \mathrm{rpm}$ for $24 \mathrm{~h}$ at $25^{\circ} \mathrm{C} .15 \mathrm{ml}$ fermented sample was centrifuged at $10000 \mathrm{rpm}$ for $10 \mathrm{~min}$ at $4^{\circ} \mathrm{C}$. Pellet was collected. Two phases separation method was used in prodigiosin extraction. Chloroform and sodium hypochlorite was used in 1:1 and extracted pigment was collected in chloroform. Optical density of the resulting solution was determined at $535 \mathrm{~nm}$ (OD $535 \mathrm{~nm}$ ). The total prodigiosin $(\mathrm{mg} / \mathrm{l})$ was calculated according to the following formula (Williams and Gott, 1961; Chen et al., 2006).

$$
\mathrm{TP} \mathrm{mg} / \mathrm{l}=\frac{\mathrm{ADV} 1}{7.07 \times 10^{4} \mathrm{~V} 2}
$$

where TP denotes the total pigment yield ( $\mathrm{mg} / \mathrm{l})$, A the absorbance of chloroform extract at $535 \mathrm{~nm}$, D the dilution ratio, $\mathrm{V}_{1}$ the volume of methanol added, $7.07 \times 10^{4}$ is extinction coefficient of prodigiosin and $\mathrm{V}_{2}$ is the volume of fermentative liquid.

\section{Purification of prodigiosin using TLC}

The crude pigment extracted from solid-state fermentation broth after $96 \mathrm{~h}$ of cultivation. After extraction, pigment was further purified using preparative thin layer chromatography (TLC) $(2.5 \times 30 \mathrm{~cm}$; Kieselgel 60; Merck, Darmstadt, Germany) mesh size: $60-80$. Thin layer chromatography was used for separation of the non-coloured impurity (Montaner and Pérez, 2002). The solvent system used for the TLC was chloroform: methanol (5:5) (Figure 2) Spots of pigment refined by TLC were scratched and 
separated in chloroform. The extract was transferred into neat and clean vials and then centrifuge in $12000 \mathrm{rpm}$ for $15 \mathrm{~min}$ to separate silica and pure red pigment. After separation, supernatant was collected which contained purified pigment. Purified supernatant transfer in porcelain dissipating dishes and evaporated at room temperature till a powdered pigment was gotten.

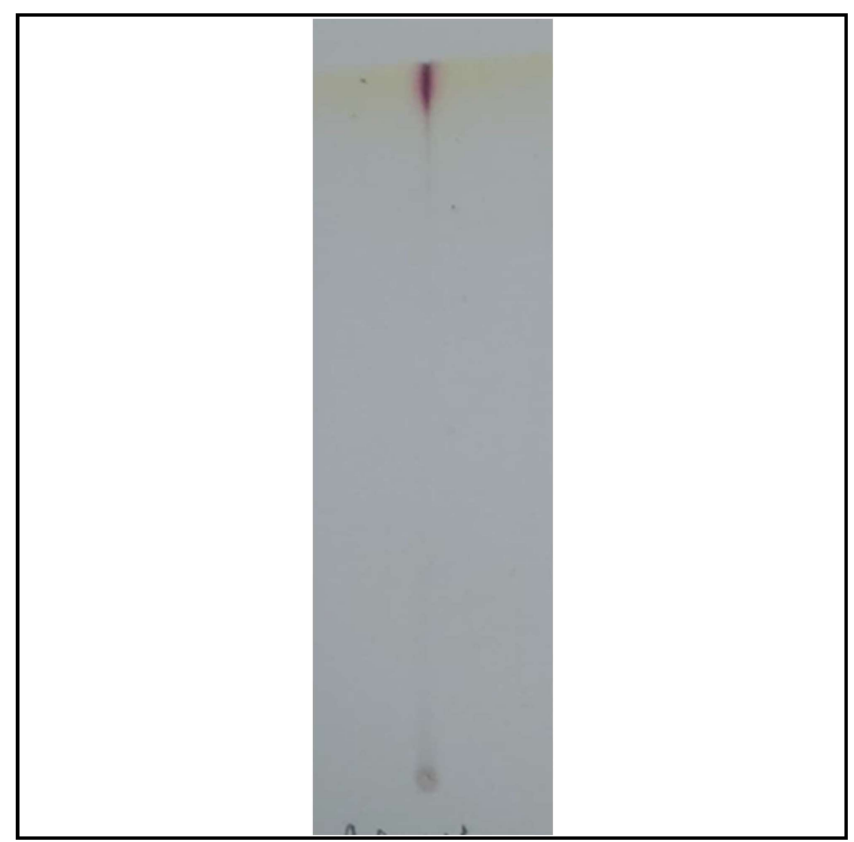

Figure 2: Thin layer chromatography of extracted pigment.

\section{Results}

4.1 Screening of suitable substrate for enhanced prodigiosin yield

The pigment yield is significantly affected by type of substrate and its amount, fermentation, chosen strain, inoculum size and moisture percentage. In the present study, we investigated the effect of agroindustrial waste on pigment yield under SSF. Serratia nematodiphilia (NCIM 5606) was grown in different media (wheat bran, sweet lemon orange peel, pigeon pea and rice bran). Wheat bran showed maximum prodigiosin yield of $1.3075 \mathrm{mg} / \mathrm{l}$ after $96 \mathrm{~h}$ of incubation (Table 1). Similarly, Serratia nematodiphilia grown on sweet lemon and orange peel media gave prodigiosin yield of 0.1693 and $0.1495 \mathrm{mg} / \mathrm{l}$, respectively. Minimum prodigiosin yield was observed in SSF comprising pigeon pea and rice grain as substrate (Table 1).

Table 1: Production of prodigiosin in different substrate in solid state fermentation

\begin{tabular}{|l|l|l|}
\hline Sr. No. & Substrate & Yield mg/l \\
\hline 1 & Wheat bran & 1.3075 \\
2 & Sweet lemon & 0.1693 \\
3 & Orange peel & 0.1495 \\
4 & Pigeon pea & 0.0082 \\
5 & Rice bran & 0.0667 \\
\hline
\end{tabular}

\subsection{Purification of prodigiosin}

The extracted purified red pigment showed Rf value of 0.85 which was comparable to standard prodigiosin (Figure 2). This was in correlation with previous findings of Pathak and Dharmadhikari (2016) who reported Rf values of 0.9 for prodigiosin. Similarly, Raj et al. (2009), reported Rf value of 0.9 for the prodigiosin extracted from Serratia Marcescens MTCC 97 under similar condition.

\subsection{Presumption test for extracted pigment}

Presumption test was performed for the confirmation of prodigiosin. Pellet was tested against acidic and alkaline conditions. In acidic condition, red or pink color was obtained and alkaline condition yellow or tan color obtained, that was confirmed a positive presumptive test for prodigiosin.

\subsection{Characterization of red pigment \\ 4.4.1 UV-vis spectral analysis}

The absorbance spectrum of sample was measured with a Double Beam UV-Visible Spectrophotometer (Electronics India model No3375 ) in the range of 350 to $700 \mathrm{~nm}$. Absorption spectra of the red pigment in chloroform showed a maximum absorbance at $535 \mathrm{~nm}$ evidenced by the presence of a peak at this wave length (Figure 3). A single peak absorbance at $535 \mathrm{~nm}$ depicted the extracted pigment as prodigiosin which was in conformity with previous findings (Giri et al., 2004; Montaner et al., 2000).

\subsection{Characterization of prodigiosin}

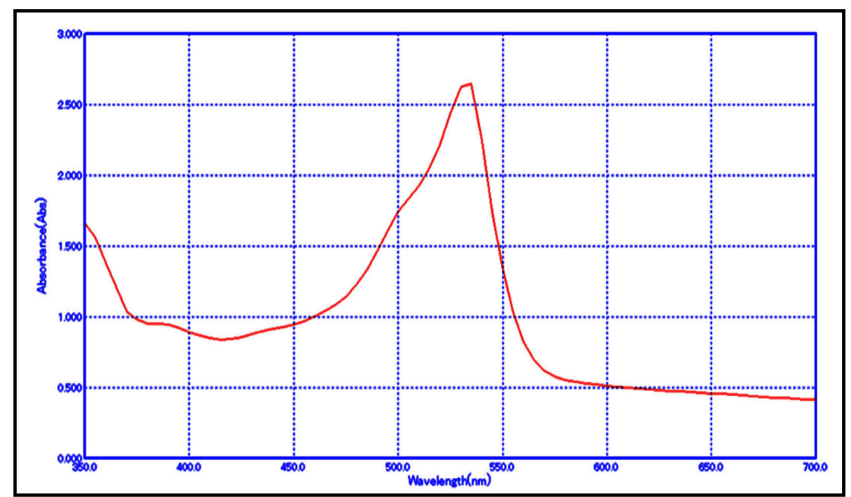

Figure 3: Absorbance spectra of Prodigiosin (535 nm).

\subsubsection{Fourier transform infrared spectrum of pigmented metabolite}

Purified pigment was characterized by FTIR as prodigiosin. FTIR spectra showed peaks at wavenumber; $2960.17,1718.97,1444.96$, $1381.26,1282.34,1214.97,1122.85,1073.58,939.68,749.59$ and $666.95 \mathrm{~cm}^{-1}$. The peaks at $2960.17 \mathrm{~cm}^{-1}$ are due to asymmetrical and symmetrical stretching of methylene groups. The peaks at $1718.97 \mathrm{~cm}^{-1}$ and $1445 \mathrm{~cm}^{-1}$ are due to the presence of $-\mathrm{NH}$ and methyl groups. The visible peak at $1381.26 \mathrm{~cm}^{-1}$ is due to the presence of C-O group in prodigiosin. The fingerprint region for the red pigment was characterized by medium intensity bands at 1073.58, (C-O and C-N) and $939.68 \mathrm{~cm}^{-1}$. The peaks around 1282.34 $\mathrm{cm}^{-1}$ and $749.59 \mathrm{~cm}^{-1}$ are attributed to carbon-carbon double bond. The characteristic peaks observed at specific wavenumbers deduced 
the extracted pigment as prodigiosin (Figure 4). Similar findings were reported by Faraag et al. (2017), in FTIR study for characterization of prodigiosin produced by Serratia marcescens strain isolated from irrigation water in Egypt.

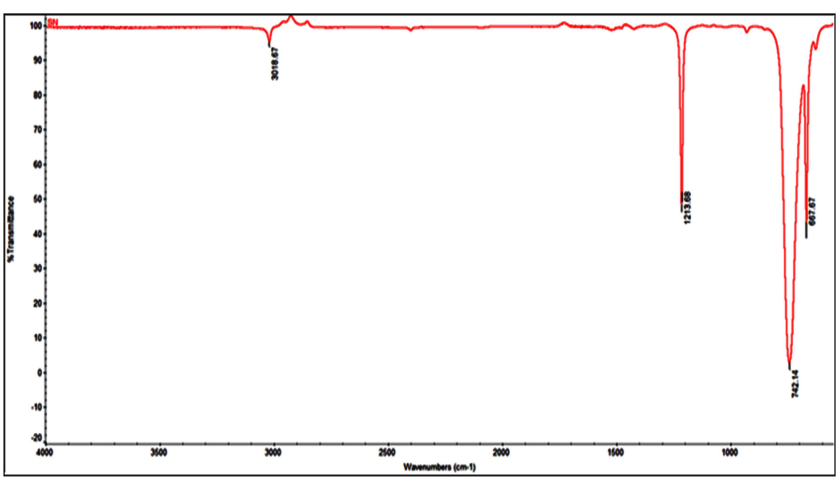

Figure 4: Fourier transform infra-red spectrum of purified prodigiosin.

\section{Discussion}

The present study provides an evidence for the successfully utilization of agroindustrial waste to enhance prodigiosin production under solid-state fermentation. Serratia nematodiphilia (NCIM 5606) was grown in different media (wheat bran, sweet lemon orange peel, pigeon pea and rice bran). But, wheat bran was best substrate for production of prodigiosin in all of them. Results revealed that maximum production of prodigiosin was obtained in wheat bran medium $(1.3075 \mathrm{mg} / \mathrm{l})$. Prodigiosin production is totally dependent on nitrogenous substance. Many substrate contain different type of amino acid, but they could not help the production of prodigiosin because specific amino acid utilize in production of prodigiosin. Wheat bran contains 5 types of specific amino acids (alanine, proline, leucine, glycine, cysteine), which was necessary for enhance production, they have showed increase in the production of prodigiosin when using the proline resulted in yield $(17.3 \mathrm{mg} / \mathrm{l})$ (Mathlom et al., 2018). Wheat bran contains elevated level of these amino acids and gives better prodigiosin yield (Balakrishnan, 1996). Maximum prodigiosin yield of $270 \mathrm{mg} / \mathrm{l}$ was observed in MO-1 using ram horn peptone as sole nitrogen source under SMF (Kurbanoglu et al., 2015).

Extracted purified pigment show Rf value which was similar to previous findings by Pathak and Dharmadhikari. (2016). So, this was prove that pigment was prodigiosin.

Presumptive test was performed for confirmation of prodigiosin. In acidic condition, prodigiosin was red in color and alkaline condition pigment show yellow or tan color. This presumptive test proved that pigment was prodigiosin.

The absorbance spectrum of sample show the presence of specific chemical in solution. In sample maximum absorbance obtained $535 \mathrm{~nm}$. Maximum absorbance indicate the presence of prodigiosin in extracted sample. A single peak absorbance at $535 \mathrm{~nm}$ depicted the extracted pigment as prodigiosin which was in conformity with previous findings (Giri et al., 2004; Montaner et al., 2000).
FTIR analysis of crude pigment shows the presence of prodigiosin as active compound. The characteristic peaks observed at specific wavenumbers deduced the extracted pigment as prodigiosin. Figure 4 depicts the different FTIR band and similar findings were reported by Faraag et al. (2017), in FTIR study for characterization of prodigiosin produced by Serratia marcescens strain isolated from irrigation water in Egypt. So, all discussion proved that extracted pigment was prodigiosin.

\section{Conclusion}

Serratia nematodiphilia gave a maximum pigment yield of in solid-state fermentation using wheat bran as a substrate. The pigment yield observed in present investigation under solid-state fermentation showed 5 fold better yield in comparison to control. Further, characterisation of extracted pigment by absorption spectra and FTIR confirmed the presence of prodigiosin as a bioactive metabolite. The present investigation deduced that wheat bran can be effectively used as primary substrate in prodigiosin production at commercial level. The usage of agroindustrial waste will minimize the upstream processing cost of novel pigment and can be effectively used in food processing industries as food color. Further, the process may be scale up for its commercial application at industrial level.

\section{Acknowledgments}

This study was financially supported by National Fellowship for Other Backward Classes by University Grant Commission, New Delhi, India. The authors gratefully acknowledge School of Biochemical Engineering, Indian Institute of Technology (BHU), Varanasi, India for providing chemicals and infrastructure facilities for this study. The authors also acknowledge the contribution of Central Instrumentation Laboratory (CIL), Indian Institute of Technology for FTIR f acility.

\section{Conflict of interest}

The authors declare that there are no conflicts of interest in the course of conducting the research. Both the authors had final decision regarding the manuscript and decision to submit the findings for publication.

\section{References}

Balakrishnan, K. (1996). Production of biologically active secondary metabolites in solid state fermentation. Journal of Scientific and Industrial Research, 55:365-372.

Boger, D.L. and Patel, M. (1988). Total synthesis of prodigiosin, prodigiosene, and desmethoxyprodigiosin: Diels-Alder reactions of heterocyclic azadienes and development of an effective palladium (II)-promoted 2, 2'-bipyrrole coupling procedure. The Journal of Organic Chemistry, 53(7):1405-1415.

Cerdeño, A.M.; Bibb, M.J. and Challis, G.L. (2001). Analysis of the prodiginine biosynthesis gene cluster of Streptomyces coelicolor A3 (2): New mechanisms for chain initiation and termination in modular multienzymes. Chemistry and Biology, 8(8):817-829.

Chen, D.; Han, Y. and Gu, Z. (2006). Application of statistical methodology to the optimization of fermentative medium for carotenoids production by Rhodobacter sphaeroides. Process Biochemistry, 41(8): 1773-1778. 
Duenas, R.; Tengerdy, R.P. and Gutierrez-Correa, M. (1995). Cellulase production by mixed fungi in solid-substrate fermentation of bagasse. World Journal of Microbiology and Biotechnology, 11(3):333-337.

Downham, A. and Collins, P. (2000). Colouring our foods in the last and next millennium. International Journal of Food Science and Technology, 35(1):5-22.

Faraag, A.H.; El-Batal, A.I. and El-Hendawy, H.H. (2017). Characterization of prodigiosin produced by Serratia marcescens strain isolated from irrigation water in Egypt. Nature and Science, 15:55-68.

Frustner, A. (2003). Chemistry and Biology of roseopnium and the prodigiosin alkoids: A survey of the last 2500 years. Angewandte Chemie International Edition, 42:3582-3603.

Giri, A.V.; Anandkumar, N.; Muthukumaran, G. and Pennathur, G. (2004). A novel medium for the enhanced cell growth and production of prodigiosin from Serratia marcescens isolated from soil. BMC Microbiology, 4(1): 11 .

Kobayashi, N. and Ichikawa, Y. (1991). Separation of the prodigiosinlocalizing crude vesicles which retain the activity of protease and nuclease in Serratia marcescens. Microbiology and Immunology, 35(8):607-614.

Kurbanoglu, E.B.; Ozdal, M.; Ozdal, O.G. and Algur, O. F. (2015). Enhanced production of prodigiosin by Serratia marcescens MO-1 using ram horn peptone. Brazilian Journal of Microbiology, 46(2):631-637.

Mathlom, G.S.; Hayder, N.H. and Mahmood, M.S. (2018). Synergistic effect of biosurfactant and prodigiosin produced by Serratia marcescens as antimicrobial agent. Current Research in Microbiology and Biotechnology, 6(2):1601-1615.

Matsuyama, T.; Murakami, T.; Fujita, M.; Fujita, S. and Yano, I. (1986) Extracellular vesicle formation and biosurfactant production by Serratia marcescens. Microbiology, 132(4):865-875.

Montaner, B. and Pérez-Tomás, R. (2002). The cytotoxic prodigiosin induces phosphorylation of $\mathrm{p} 38$-MAPK but not of SAPK/JNK. Toxicology Letters, 129(1-2):93-98.
Montaner, B.; Navarro, S.; Piqué, M.; Vilaseca, M.; Martinell, M.; Giralt, E. and Pérez Tomás, R. (2000). Prodigiosin from the supernatant of Serratia marcescens nduces apoptosis in haematopoietic cancer cell lines. British Journal of Pharmacology, 131(3):585-593.

Murugesan, K.; Nam, I.H.; Kim, Y.M. and Chang, Y.S. (2007). Decolorization of reactive dyes by a thermostable laccase produced by Ganoderma lucidum in solid state culture. Enzyme and Microbial Technology, 40(7):1662-1672.

Phatake, Y.B. and Dharmadhikari, S.M. (2016). Isolation and screening of prodigiosin production bacteria and characterization of produced pigment. International Journal of Science and Nature, 7(1):202209.

Raj, D.N.; Dharumaduari, D.; Noorudin, T. and Annamalai, P. (2009). Production of prodigiosin from Serratia marcescens and its cytotoxicity activity. Journal of Pharmacy Research, 2(4):590-593.

Rymbai, H.; Sharma, R.R. and Srivastav, M. (2011). Bio-colorants and its implications in health and food industry: A review. International Journal of Pharmacological Research, 3(4):2228-2244.

Samrot, A.V.; Chandana, K.; Senthilkumar, P. and Narendra, K.G. (2011). Optimization of prodigiosin production by Serratia marcescens SU-10 and evaluation of its bioactivity. International Research Journal of Biotechnology, 2(5):128-133.

Sathya, R.; Pradeep, B.V.; Angayarkanni, J. and Palaniswamy, M. (2009). Production of milk clotting protease by a local isolate of Mucor circinelloides under SSF using agro-industrial wastes. Biotechnology and Bioprocess Engineering, 14(6):88-794.

Sharma, D. (2014). Understanding biocolour: A review. International Journal of Scientific and Technology Research, 3:294-299.

Williams, R.P.; Gott, C.L. and Green, J.A. (1961). Studies on pigmentation of Serratia marcescens V.: Accumulation of pigment fractions with respect to length of incubation time. Journal of Bacteriology, 81(3):376

Citation Kamlesh Kumar Maurya, Abhishek Dutt Tripathi, Deepak Kumar and Suresh Kumar Srivastava (2020). Production, purification and characterization of prodigiosin by Serratia nematodiphilia (NCIM 5606) using solid-state fermentation with various substrate. Ann. Phytomed., 9(2):302-306. http://dx.doi.org/10.21276/ap.2020.9.2.30 